Bangladesh J. Sci. Res. 27(2): 155-163, 2014 (December)

\title{
JORDAN HIGHER DERIVATIONS IN PRIME $\Gamma$-RINGS
}

\author{
M. M. Rahman* and A. C. Paul ${ }^{1}$ \\ ${ }^{1}$ Department of Mathematics, Jagannath University, Dhaka, Bangladesh .
}

\begin{abstract}
The objective of this paper is to study Jordan higher derivations in prime $\Gamma$-rings. We introduce a higher derivation and a Jordan higher derivation in $\Gamma$-rings. For a 2-torsion free prime $\Gamma$-ring $M$ which satisfies the condition $a \alpha b \beta c=a \beta b \alpha c$ for all $a, b, c \in M$ and $\alpha, \beta \in \Gamma$, we prove that every Jordan higher derivation $D=\left(d_{i}\right)_{i \in N_{0}}$ of $M$ is a higher derivation of $M$.
\end{abstract}

Keywords: Higher derivation, Jordan higher derivation, prime $\Gamma$-ring.

\section{Introduction}

We begin with the general definition of a $\Gamma$-ring. The notion of a $\Gamma$-ring was introduced by Nobusawa (1964) and generalized by Barnes (1966) as defined below. Let $M$ and $\Gamma$ be additive abelian groups. If there is a mapping $M \times \Gamma \times M \rightarrow M$ such that the conditions

- $(x+y) \alpha z=x \alpha z+y \alpha z, x(\alpha+\beta) y=x \alpha y+x \beta y, x \alpha(y+z)=x \alpha y+x \alpha z$

- $(x \alpha y) \beta z=x \alpha(y \beta z)$

are satisfied for all $x, y, z \in M, \alpha, \beta \in \Gamma$, then $M$ is called a $\Gamma$-ring. This concept is more general than that of a ring. From the definition it is clear that every ring is a $\Gamma$-ring but the converse is not necessarily true. A $\Gamma$-ring $M$ is 2-torsion free if $2 a=0$ implies $a=0$ for all $a \in M ; \quad M$ is called a prime $\Gamma$-ring if for all $a, b \in M, a \Gamma M \Gamma b=0$ implies $a=0$ or $b=0$.

The concepts of derivation and Jordan derivation of a $\Gamma$-ring have been introduced by Sapanci and Nakajima (1997). For classical ring theory, Herstien (1957) proved a well known result that every Jordan derivation of a 2-torsion free prime ring is a derivation. Bresar (1988) proved this result for semiprime rings. Sapanci and Nakajima (1997) proved the same result for completely prime $\Gamma$-rings. Haetinger (2002) worked on higher derivations on prime rings and extended this result to Lie ideals in a prime ring. In this article, we introduce a higher derivation and a Jordan higher derivation in $\Gamma$-rings. We extend the result of Cortes and Haetinger (2005) concerning Jordan higher derivations in prime $\Gamma$-rings. We prove that every Jordan higher derivation of a 2torsion free prime $\Gamma$-ring satisfying the condition $a \alpha b \beta c=a \beta b \alpha c$ for all $a, b, c \in M$ and $\alpha, \beta \in \Gamma$, is a higher derivation of $M$.

Corresponding author e-mail: <mizanorrahman@gmail.com>. 1Department of Mathmatics, University of Rajshahi, Rajshahi, Bangladesh. 
Throughout the article, we asume the condition $a \alpha b \beta c=a \beta b \alpha c$ for all $a, b, c \in M$ and $\alpha, \beta \in \Gamma$ and refer it to by $\left(^{*}\right)$.

\section{Jordan Derivations in a Prime $\Gamma$-ring}

The notions of derivation and Jordan derivation of $\Gamma$-rings have been introduced by Sapanci and Nakajima (1997) as follows.

Definition 1. For a $\Gamma$-ring $M$, if $d: M \rightarrow M$ is an additive mapping such that $d(a \alpha b)=d(a) \alpha b+a \alpha d(b)$ holds for all $a, b \in M$ and $\alpha \in \Gamma$, then $d$ is called $a$ derivation of $M ; d$ is called a Jordan derivation of $M$ if $d(a \alpha a)=d(a) \alpha a+a \alpha d(a)$ holds for all $a \in M$ and $\alpha \in \Gamma$.

First, we show that every Jordan derivation of a 2-torsion free prime $\Gamma$-ring is a derivation. For this purpose we prove the following Lemmas.

Lemma 1. Let $M$ be a $\Gamma$-ring, and let $d$ be a Jordan derivation of $M$. Then for all $a, b, c \in M$ and $\alpha, \beta \in \Gamma$, the following statements hold:

(i) $d(a \alpha b+b \alpha a)=d(a) \alpha b+d(b) \alpha a+a \alpha d(b)+b \alpha d(a)$

(ii) $\quad d(a \alpha b \beta a+a \beta b \alpha a)=d(a) \alpha b \beta a+d(a) \beta b \alpha a+a \alpha d(b) \beta a$ $+a \beta d(b) \alpha a+a \alpha b \beta d(a)+a \beta b \beta d(a)$.

In particular, if $M$ is 2-torsion free and satisfies the condition $\left(^{*}\right)$, then

(iii) $\quad d(a \alpha b \beta a)=d(a) \alpha b \beta a+a \alpha d(b) \beta a+a \alpha b \beta d(a)$

(iv) $d(a \alpha b \beta c+c \alpha b \beta a)=d(a) \alpha b \beta c+d(c) \alpha b \beta a+a \alpha d(b) \beta c$ $+c \alpha d(b) \beta a+a \alpha b \beta d(c)+c \alpha b \beta d(a)$.

Proof. Compute $d((a+b) \alpha(a+b))$ and cancel the like terms from both sides to obtain (i). Then replace $a \beta b+b \beta a$ for $b$ in (i) to get (ii). Using the condition (*), and since $M$ is 2 torsion free, (iii) follows from (ii). Finally, (iv) is obtained by replacing $a+c$ for $a$ in (iii).

Definition 2. Let $d$ be a Jordan derivation of $a \Gamma$-ring $M$. Then for all $a, b \in M$ and $\alpha \in \Gamma$, we define $\phi_{\alpha}(a, b)=d(a \alpha b)-d(a) \alpha b-a \alpha d(b)$. Thus $\phi_{\alpha}(b, a)=d(b \alpha a)-d(b) \alpha a-b \alpha d(a)$. Lemma 2. Let $d$ be a Jordan derivation of $a \Gamma-$ ring $M$. Then for all $a, b, c \in M$ and $\alpha, \beta \in \Gamma$, the following statements hold: 
(i) $\phi_{\alpha}(a, b)+\phi_{\alpha}(b, a)=0$; (ii) $\phi_{\alpha}(a+b, c)=\phi_{\alpha}(a, c)+\phi_{\alpha}(b, c)$

(iii) $\phi_{\alpha}(a, b+c)=\phi_{\alpha}(a, b)+\phi_{\alpha}(a, c)$; (iv) $\phi_{\alpha+\beta}(a, b)=\phi_{\beta}(a, b)+\phi_{\beta}(a, b)$.

Proof. Obvious.

Remmark 1. $d$ is a derivation of $a \Gamma$-ring $M$ if and only if $\phi_{\alpha}(a, b)=0$ for all $a, b \in M$ and $\alpha \in \Gamma$.

Lemma 3. Let $M$ be a 2-torsion free $\Gamma$-ring satisfying the condition(*), and let $d$ be a Jordan derivation of $M$. Then $\phi_{\alpha}(a, b) \beta m \gamma[a, b]_{\alpha}+[a, b]_{\alpha} \beta m \gamma \phi_{\alpha}(a, b)=0$ for all $a, b, m \in M$ and $\alpha, \beta, \gamma \in \Gamma$.

Proof. For any $a, b, m \in M$ and $\alpha, \beta, \gamma \in \Gamma$, by using Lemma 1(iv), we have

$$
\begin{aligned}
d(a \alpha b \beta m \gamma b \alpha a+b \alpha a \beta m \gamma a \alpha b)= & d((a \alpha b) \beta m \gamma b \alpha a+(b \alpha a) \beta m \gamma(a \alpha b)) \\
= & d(a \alpha b) \beta m \gamma b \alpha a+a \alpha b \beta d(m) \gamma b \alpha a+a \alpha b \beta m \gamma d(b \alpha a), \\
& +d(b \alpha a) \beta m \gamma a \alpha b+b \alpha a \beta d(m) \gamma a \alpha b+b \alpha a \beta m \gamma d(a \alpha b)
\end{aligned}
$$

On the other hand, by using Lemma 1 (iii)

$$
\begin{aligned}
& d(a \alpha(b \beta m \gamma b) \alpha a+b \alpha(a \beta m \gamma a) \alpha b)=d(a \alpha(b \beta m \gamma b) \alpha a)+d(b \alpha(a \beta m \gamma a) \alpha b) \\
& =d(a) \alpha b \beta m \gamma b \alpha a+a \alpha d(b \beta m \gamma b) \alpha a+a \alpha b \beta m \gamma b \alpha d(a) \\
& +d(b) \alpha a \beta m \gamma a \alpha b+b \alpha d(a \beta m \gamma a) \alpha b+b \alpha a \beta m \gamma a \alpha d(b) \\
& =d(a) \alpha b \beta m b a a+a a d(b) \beta m b a a+a d b \beta d(m)\} b a a \\
& +a d b \beta m d(b) a a+a d b \beta m b a d(a)+d(b) a a \beta m a a d b+b a d(a) \\
& \beta m a a b+b a a \beta d(m) \gamma a c b+b a a \beta m d(a) c b+b a a \beta m a a d(b) .
\end{aligned}
$$

Comparing the two relations and using the Definition 2, we obtain

$$
\phi_{\alpha}(a, b) \beta m \gamma b \alpha a+\phi_{\alpha}(b, a) \beta m \gamma a \alpha b+a \alpha b \beta m \gamma \phi_{\alpha}(b, a)+b \alpha a \beta m \gamma \phi_{\alpha}(a, b)=0 .
$$

This implies that

$$
\phi_{\alpha}(a, b) \beta m \gamma[a, b]_{\alpha}+[a, b]_{\alpha} \beta m \gamma \phi_{\alpha}(a, b)=0, \forall a, b, m \in M \text { and } \alpha, \beta, \gamma \in \Gamma
$$

Lemma 4. Let $M$ be a 2-torsion free prime $\Gamma$-ring and let $a, b \in M$.

If $a \alpha m \beta b+b \alpha m \beta a=0$ for all $m \in M, \alpha, \beta \in \Gamma$, then $a=0$ or $b=0$. 
Proof. Replacing $m$ by $s \delta a \mu t$ in $a \alpha m \beta b+b \alpha m \beta a=0$, we have $a \alpha s \delta a \mu t \beta b+b \alpha s \delta a \mu t \beta a=0$.

Now $b \alpha s \delta a=-a \alpha s \delta b$ and $a \mu t \beta b=-b \mu t \beta a$. Substituting these we get

$$
\begin{aligned}
-a \alpha s \delta b \mu t \beta a-a \alpha s \delta b \mu t \beta a & =0 . \\
\Rightarrow 2 a \alpha s \delta b \mu t \beta a & =0 .
\end{aligned}
$$

As $M$ is 2-torsion free, so $a \alpha s \delta b \mu t \beta a=0$.

Therefore, $(a \alpha s \delta b) \Gamma M \Gamma a=0$. As $M$ is prime, so $a \alpha s \delta b=0$ or $a=0$.

Suppose $a \alpha s \delta b=0$. Again applying the primeness of $M$, we have $a=0$ or $b=0$.

Theorem 1. Let $M$ be a 2-torsion free prime $\Gamma$-ring satisfying the condition (*), and let $d$ be a Jordan derivation of $M$. Then $d$ is a derivation of $M$.

Proof. By Lemma 3 and Lemma 4, and $M$ being prime, we have

$\phi_{\alpha}(a, b)=0$ or $[a, b]_{\alpha}=0$.

If $[a, b]_{\alpha}=0$ for all $a, b \in M, \alpha \in \Gamma$, then $a \alpha b=b \alpha a$. Using this in Lemma 1(i), we have $2 d(a \alpha b)=2 d(a) a b+2 a a d(b)$. Since $M$ is 2-torsion free, we obtain $d$ is a derivation of $M$.

If $\phi_{\alpha}(a, b)=0$, then $d$ is also a derivation of $M$.

\section{Jordan Higher Derivations in Prime $\Gamma$-Rings}

We introduce higher derivation and Jordan higher derivation of $\Gamma$-rings in the following way.

Definition 3. Let $D=\left(d_{i}\right)_{i \in N_{0}}$ be a family of additive mappings of a $\Gamma$ - ring $M$ such that $d_{0}=i d_{M}$, where $i d_{M}$ is an identity mapping on $M$ and $N_{0}=N \cup\{0\}$. Then $D$ is a higher derivation of $M$ if for each $n \in N_{0}$ and $i, j \in N_{0}$,

$$
d_{n}(a \alpha b)=\sum_{i+j=n} d_{i}(a) \alpha d_{j}(b), \text { holds for all } a, b \in M ; \alpha \in \Gamma,
$$

$D$ is a Jordan higher derivation of $M$ if

$$
d_{n}(a \alpha a)=\sum_{i+j=n} d_{i}(a) \alpha d_{j}(a) \text {, holds for all } a \in M ; \alpha \in \Gamma \text {. }
$$


Example 1. Let $R$ be an associative ring with 1 . Let us consider $M=M_{1,2}(R)$ and $\Gamma=\left\{\left(\begin{array}{c}n .1 \\ 0\end{array}\right): n \in Z\right\}$, then $M$ is a $\Gamma$-ring. Let $f_{n}: R \rightarrow R$ be a higher derivation for each $n \in N_{0}$. For $n \in N_{0}$, we define additive mappings $d_{n}: M \rightarrow M$ by $d_{n}((a, b))=\left(f_{n}(a), f_{n}(b)\right)$. Then an easy verifications leads to us that $d_{n}$ is a higher derivation of $M$. Let $P=\{(a, a): a \in R\}$, then $P$ is a $\Gamma$-ring contained in $M$. In fact, $P$ is a sub $\Gamma$-ring. Define $d_{n}((a, a))=\left(f_{n}(a), f_{n}(a)\right)$, then $d_{n}$ is a Jordan higher derivation of $P$.

Lemma 5. Assume that $D=\left(d_{i}\right)_{i \in N}$ is a Jordan higher derivation of $M$. Then for all $a, b, c \in M ; \alpha, \beta \in \Gamma$ and $n \in N$,

(i) $d_{n}(a \alpha b+b \alpha a)=\sum_{i+j=n}\left[d_{i}(a) \alpha d_{j}(b)+d_{i}(b) \alpha d_{j}(a)\right]$;

(ii) $d_{n}(a \alpha b \beta a)=\sum_{i+j+k=n}\left[d_{i}(a) \alpha d_{j}(b) \beta d_{k}(a)\right]$;

(iii) $d_{n}(a \alpha b \beta c+c \alpha b \beta a)=\sum_{i+j+k=n}\left[d_{i}(a) \alpha d_{j}(b) \beta d_{k}(c)+d_{i}(c) \alpha d_{j}(b) \beta d_{k}(a)\right]$.

Proof. The proofs of (i) and (ii) are similar to the proofs of Lemma 1(i) and Lemma 1(iii). Replacing $a$ by $a+c$ in (ii) and using (ii), we obtain

$$
\begin{gathered}
W=d_{n}((a+c) \alpha b \beta(a+c))=\sum_{i+j+k=n} d_{i}(a+c) \alpha d_{j}(b) \beta d_{k}(a+c) \\
=\sum_{i+j+k=n}\left(d_{i}(a)+d_{i}(c)\right) \alpha d_{j}(b) \beta\left(d_{k}(a)+d_{k}(c)\right)=\sum_{i+j+k=n} d_{i}(a) \alpha d_{j}(b) \beta d_{k}(a) \\
+\sum_{i+j+k=n} d_{i}(a) \alpha d_{j}(b) \beta d_{k}(c)+\sum_{i+j+k=n} d_{i}(c) \alpha d_{j}(b) \beta d_{k}(a)+\sum_{i+j+k=n} d_{i}(c) \alpha d_{j}(b) \beta d_{k}(c) .
\end{gathered}
$$

Also, we have

$$
\begin{aligned}
& W=d_{n}(a \alpha b \beta a+a \alpha b \beta c+c \alpha b \beta a+c \alpha b \beta c) \\
& =d_{n}(a \alpha b \beta a)+d_{n}(c \alpha b \beta c)+d_{n}(a \alpha b \beta c+c \alpha b \beta a) \\
& =\sum_{i+j+k=n} d_{i}(a) \alpha d_{j}(b) \beta d_{k}(a)+\sum_{i+j+k=n} d_{i}(c) \alpha d_{j}(b) \beta d_{k}(c)+d_{n}(a \alpha b \beta c+c \alpha b \beta a) .
\end{aligned}
$$

By comparing the two expressions for $W$, we obtain (iii). 
Definition 4 For any Jordan higher derivation $D=\left(d_{i}\right)_{i \in N}$ of $M$, we define $\phi_{n}^{\alpha}(a, b)=d_{n}(a \alpha b)-\sum_{i+j=n} d_{i}(a) \alpha d_{j}(b)$ for all $a, b \in M ; \alpha \in \Gamma$ and $n \in N$.

Remmark 2. $D$ is a higher derivation of $M$ if and only if $\phi_{n}^{\alpha}(a, b)=0$ holds for all $a, b \in M ; \alpha \in \Gamma$ and $n \in N$.

Lemma 6. For every $a, b, c \in M ; \alpha, \beta \in \Gamma$ and $n \in N$,
(i) $\phi_{n}^{\alpha}(a, b)+\phi_{n}^{\alpha}(b, a)=0$;
(ii) $\phi_{n}^{\alpha}(a+b, c)=\phi_{n}^{\alpha}(a, c)+\phi_{n}^{\alpha}(b, c)$

(iii) $\phi_{n}^{\alpha}(a, b+c)=\phi_{n}^{\alpha}(a, b)+\phi_{n}^{\alpha}(a, c)$;

(iv) $\phi_{n}^{\alpha+\beta}(a, b)=\phi_{n}^{\alpha}(a, b)+\phi_{n}^{\beta}(a, b)$.

Proof. (i) By Definition 4 and using Lemma 5(i), we obtain

$$
\begin{aligned}
\phi_{n}^{\alpha}(a, b)+\phi_{n}^{\alpha}(b, a) & =d_{n}(a \alpha b)-\sum_{i+j=n} d_{i}(a) \alpha d_{j}(b)+d_{n}(b \alpha a)-\sum_{i+j=n} d_{i}(b) \alpha d_{j}(a) \\
& =d_{n}(a \alpha b+b \alpha a)-\sum_{i+j=n} d_{i}(a) \alpha d_{j}(b)-\sum_{i+j=n} d_{i}(b) \alpha d_{j}(a) \\
& =\sum_{i+j=n} d_{i}(a) \alpha d_{j}(b)+\sum_{i+j=n} d_{i}(b) \alpha d_{j}(a)-\sum_{i+j=n} d_{i}(a) \alpha d_{j}(b) \\
& -\sum_{i+j=n} d_{i}(b) \alpha d_{j}(a)=0 .
\end{aligned}
$$

(ii) By Definition 4, we get

$$
\begin{aligned}
\phi_{n}^{\alpha}(a+b, c) & =d_{n}((a+b) \alpha c)-\sum_{i+j=n} d_{i}(a+b) \alpha d_{j}(c) \\
& =d_{n}(a \alpha c+b \alpha c)-\sum_{i+j=n} d_{i}(a) \alpha d_{j}(c)-\sum_{i+j=n} d_{i}(b) \alpha d_{j}(c) \\
& =d_{n}(a \alpha c)-\sum_{i+j=n} d_{i}(a) \alpha d_{j}(c)+d_{n}(b \alpha c)-\sum_{i+j=n} d_{i}(b) \alpha d_{j}(c) \\
& =\phi_{n}^{\alpha}(a, c)+\phi_{n}^{\alpha}(b, c) .
\end{aligned}
$$

(iii)-(iv): The proofs are straight forward.

Lemma 7. Suppose $D=\left(d_{i}\right)_{i \in N}$ is a Jordan higher derivation of a $\Gamma$-ring $M$. Let $n \in N$ and assume that $a, b \in M ; \alpha, \beta, \gamma \in \Gamma$. If $\phi_{m}^{\alpha}(a, b)=0$, for every $m<n$, then

$$
\phi_{n}^{\alpha}(a, b) \beta w \gamma[a, b]_{\alpha}+[a, b]_{\alpha} \beta w \gamma \phi_{n}^{\alpha}(a, b)=0 \text {, for every } w \in M .
$$


Proof. We consider $G=d_{n}(a \alpha b \beta w \gamma b \alpha a+b \alpha a \beta w \gamma a \alpha b)$. First, we compute

$$
G=d_{n}(a \alpha(b \beta w \gamma b) \alpha a)+d_{n}(b \alpha(a \beta w \gamma a) \alpha b) .
$$

Using Lemma 5(ii), we have on one hand

$$
\begin{gathered}
G=\sum_{i+p+l=n} d_{i}(a) \alpha d_{p}(b \beta w \gamma b) \alpha d_{l}(a)+\sum_{i+p+l=n} d_{i}(b) \alpha d_{p}(a \beta w \gamma a) \alpha d_{l}(b) \\
=\sum_{i+j+k+h+l=n} d_{i}(a) \alpha d_{j}(b) \beta d_{k}(w) \gamma d_{h}(b) \alpha d_{l}(a)+\sum_{i+j+k+h+l=n} d_{i}(b) \alpha d_{j}(a) \beta d_{k}(w) \gamma d_{h}(a) \alpha d_{l}(b) .
\end{gathered}
$$

On the other hand

$$
G=d_{n}((a \alpha b) \beta w \gamma(b \alpha a)+(b \alpha a) \beta w \gamma(a \alpha b)) .
$$

Using Lemma 5(iii), we obtain

$$
\begin{aligned}
& G=\sum_{r+s+t=n}\left(d_{r}(a \alpha b) \beta d_{s}(w) \gamma d_{t}(b \alpha a)+d_{r}(b \alpha a) \beta d_{s}(w) \gamma d_{t}(a \alpha b)\right) \\
& =\sum_{r+s+t=n} d_{r}(a \alpha b) \beta d_{s}(w) \gamma d_{t}(b \alpha a)+\sum_{r+s+t=n} d_{r}(b \alpha a) \beta d_{s}(w) \gamma d_{t}(a \alpha b) .
\end{aligned}
$$

Comparing the two expressions for $G$, we obtain

$$
\begin{aligned}
& \sum_{i+j+k+h+l=n} d_{i}(a) \alpha d_{j}(b) \beta d_{k}(w) \gamma d_{h}(b) \alpha d_{l}(a)-\sum_{r+s+t=n} d_{r}(a \alpha b) \beta d_{s}(w) \gamma d_{t}(b \alpha a) \\
+ & \sum_{i+j+k+h+l=n} d_{i}(b) \alpha d_{j}(a) \beta d_{k}(w) \gamma d_{h}(a) \alpha d_{l}(b)-\sum_{r+s+t=n} d_{r}(b \alpha a) \beta d_{s}(w) \gamma d_{t}(a \alpha b)=0 .
\end{aligned}
$$

By the inductive assumption we can put $d_{r}(x \alpha y)$ for $\sum_{i+j=r} d_{i}(x) \alpha d_{j}(y)$, when $r<n$. Therefore,

$$
\begin{aligned}
& \sum_{i+j+k+h+l=n} d_{i}(a) \alpha d_{j}(b) \beta d_{k}(w) \gamma d_{h}(b) \alpha d_{l}(a)-\sum_{r+s+t=n} d_{r}(a \alpha b) \beta d_{s}(w) \gamma d_{t}(b \alpha a) \\
= & \left(\sum_{i+j=n} d_{i}(a) \alpha d_{j}(b)\right) \beta w \gamma \beta \alpha a+a \alpha b \beta w \gamma\left(\sum_{h+l=n} d_{h}(b) \alpha d_{l}(a)\right) \\
+ & \sum_{i+j+k+h+l=n}^{i+j<n+h+l<n} d_{i}(a) \alpha d_{j}(b) \beta d_{k}(w) \gamma d_{h}(b) \alpha d_{l}(a)-d_{n}((a \alpha b) \beta w \gamma(b \alpha a) \\
- & (a \alpha b) \beta w \gamma d_{n}(b \alpha a)-\sum_{r+s+t=n}^{i+j=r<n, p+q=t<n} d_{i}(a) \alpha d_{j}(b) \beta d_{s}(w) \gamma d_{p}(b) \alpha d_{q}(a)
\end{aligned}
$$




$$
\begin{aligned}
&=-\left(d_{n}\left((a \alpha b)-\sum_{i+j=n} d_{i}(a) \alpha d_{j}(b)\right) \beta(w \gamma b \alpha a)-(a \alpha b \beta w) \gamma\left(d_{n}(b \alpha a)-\sum_{h+l=n} d_{h}(b) \alpha d_{l}(a)\right)\right. \\
&=-\left(\phi_{n}^{\alpha}(a, b) \beta w \gamma b \alpha a+a \alpha b \beta w \gamma \phi_{n}^{\alpha}(b, a)\right) .
\end{aligned}
$$

Similarly,

$$
\begin{aligned}
& \sum_{i+j+k+h+l=n} d_{i}(b) \alpha d_{j}(a) \beta d_{k}(w) \gamma d_{h}(a) \alpha d_{l}(b)-\sum_{r+s+t=n} d_{r}(b \alpha a) \beta d_{s}(w) \gamma d_{t}(a \alpha b) \\
& =-\left(\phi_{n}^{\alpha}(b, a) \beta w \gamma a \alpha b+b \alpha a \beta w \gamma \phi_{n}^{\alpha}(a, b)\right) .
\end{aligned}
$$

Hence, by using (2) and (3) in (1), we get

$$
\phi_{n}^{\alpha}(a, b) \beta w \gamma b \alpha a+a \alpha b \beta w \gamma \phi_{n}^{\alpha}(b, a)+\phi_{n}^{\alpha}(b, a) \beta w \gamma a \alpha b+b \alpha a \beta w \gamma \phi_{n}^{\alpha}(a, b)=0 .
$$

By Lemma 6(i), we have

$$
\phi_{n}^{\alpha}(a, b) \beta w \gamma b \alpha a-a \alpha b \beta w \gamma \phi_{n}^{\alpha}(a, b)-\phi_{n}^{\alpha}(a, b) \beta w \gamma a \alpha b+b \alpha a \beta w \gamma \phi_{n}^{\alpha}(a, b)=0 .
$$

This implies,

$$
\phi_{n}^{\alpha}(a, b) \beta w \gamma[a, b]_{\alpha}+[a, b]_{\alpha} \beta w \gamma \phi_{n}^{\alpha}(a, b)=0, \forall w \in M .
$$

Here, we extend the result of Cortes and Haetinger (2005) concerning Jordan higher derivations in prime $\Gamma$-rings.

Theorem 2. Let $M$ be a 2-torsion free prime $\Gamma$-ring satisfying the condition (*). Then every Jordan higher derivation of $M$ is a higher derivation of $M$.

Proof. By definition, we have

$$
\phi_{0}^{\alpha}(a, b)=0, \quad \text { for all } a, b \in M, \alpha \in \Gamma .
$$

Also, by Theorem 1 ,

$$
\phi_{1}^{\alpha}(a, b)=0, \quad \text { for all } a, b \in M, \alpha \in \Gamma \text {. }
$$

Now, we proceed by induction. Suppose that, $\phi_{m}^{\alpha}(a, b)=0$.

This implies, $d_{m}(a \alpha b)=\sum_{i+j=m} d_{i}(a) \alpha d_{j}(b)$ for all $a, b \in M ; \alpha \in \Gamma$ and $m<n$.

Taking $a, b \in M$, by Lemma 7 , we get

$$
\phi_{n}^{\alpha}(a, b) \beta w \gamma[a, b]_{\alpha}+[a, b]_{\alpha} \beta w \gamma \phi_{n}^{\alpha}(a, b)=0, \forall w \in M, \alpha, \beta, \gamma \in \Gamma .
$$

Since $M$ is prime, so by Lemma $4 \phi_{n}^{\alpha}(a, b)=0$, or $[a, b]_{\alpha}=0$. Using the similar arguments as used in the proof of Theorem 1, we obtain that every Jordan higher derivation of $M$ is a higher derivation of $M$. 


\section{References}

Barnes, W. E. 1966. On the $\Gamma$-rings of Nobusawa, Pacific J. Math. 18: 411-422.

Bresar, M. 1988. Jordan derivations on semiprime rings. Proc. Amer. Math. Soc. 104(4): 10031004.

Cortes, W. and C. Haetinger. 2005. On Jordan generalized higher derivations in rings, Turk. J. Math. 29: 1-10.

Ferrero M. and C. Haetinger. 2002. Higher derivations and a theorem by Herstein, Quaestions Mathematicae. 2(2): 249-257.

Haetinger, C. 2002. Higher derivations on Lie ideals, Tenclencias em Matematica Aplicada e. computacional, 3(1): 141-145.

Herstein, I.N. 1957. Jordan derivations of prime rings, Proc. Amer. Math. Soc., 8: 1104-1110.

Nobusawa, N. 1964. On the generalizeation of the ring theory, Osaka J. Math. 1: 81-89.

Sapanci, M. and A. Nakajima. 1997. Jordan Derivations on Completely Prime $\Gamma$-Rings, Math. Japonica. 46: 47-51.

(Manuscript received on 21 September, 2014: revised on 29 October, 2014) 